Pacific Journal of Mathematics

QUOTIENT RINGS OF RINGS WITH ZERO SINGULAR IDEAL 


\section{QUOTIENT RINGS OF RINGS WITH ZERO SINGULAR IDEAL}

\section{R. E. JoHNSON}

Many papers have been written recently (see [2]-[14] of bibliography) on extensions of rings to rings of quotients. In most of these papers, strong enough conditions are imposed on the given rings to insure that each has a vanishing singular ideal (first defined in [5]). It seems appropriate at this time to collect these results and present them in as general a form as possible. In this paper, it is assumed that each ring has a zero right singular ideal. A subsequent paper will give the quotient structure of a ring having a vanishing right and left singular ideal.

1. Introduction. If $R$ is a ring and $M$ is an $R$-module, then $L(R)$ and $L(M, R)$ will designate the lattices of right ideal of $R$ and $R$-submodules of $M$, respectively. Superscripts " $r$ " and " $l$ " will be used in designating the right and left annihilators, respectively, of an element or subset of a ring or module. The context will always make it clear from what set the annihilators are to be chosen.

In a lattice $L$ with 0 and $I$, an element $B$ is called an essential extension of element $A$, and we write $A \subset^{\prime} B$, if and only if $A \subset B$ and $C \cap A \neq 0$ for every $C$ in $L$ for which $C \cap B \neq 0$. An element $A$ of $L$ is called large if $A \subset^{\prime} I$. The sublattice of $L$ of all large elements is designated by $L^{\mathbf{\Delta}}$.

If $R$ is a ring and $M$ is a right $R$-module, then let

$$
M^{\mathbf{\Delta}}(R)=\left\{x \mid x \in M, x^{r} \in L^{\mathbf{\Delta}}(R)\right\}, \quad R^{\mathbf{\Delta}}=\left\{x \mid x \in R, x^{r} \in L^{\mathbf{\Delta}}(R)\right\} .
$$

It is easily shown that $M^{\mathbf{\Delta}}(R)$ is a submodule of $M$ and $R^{\mathbf{\Delta}}$ is a (twosided) ideal of $R$. The ideal $R^{\wedge}$ is called the singular ideal [5; p. 894] of $R$.

A ring $R$ with zero singular ideal has the unusual property, proved in [7; Section 6], that each $A \in L(R)$ has a unique maximal essential extension $A^{s}$ in $L(R)$. The mapping $s: A \rightarrow A^{s}$ of $L(R)$ is shown there to be a closure operation on $L(R)$ having the following properties:

(1) $0^{s}=0$,

(2) $(A \cap B)^{s}=A^{s} \cap B^{s}$ for each $A, B \in L(R)$, and

(3) $\left(x^{-1} A\right)^{s}=x^{-1} A^{s}$ for each $x \in R$ and $A \in L(R)$, where $x^{-1} B=\{y \mid y \in R$, $x y \in B\}$. The set $L^{s}(R)$ of closed right ideals (i.e., $A=A^{s}$ ) may be made into a lattice in the usual way by defining the union of a set of

Received December 10, 1960. This research was supported in part by a grant from the National Science Foundation. 
elements of $L^{s}(R)$ to be the least upper bound of the set. The resulting lattice $L^{s}(R)$, which is not in general a sublattice of $L(R)$, is proved to be a complete complemented modular lattice in [7; Section 6]. If $M$ is a right $R$-module for which $M^{\boldsymbol{\Delta}}(R)=0$, then the closure operation $s$ may be defined in a similar way on $L(M, R)$. The resulting lattice $L^{s}(M, R)$ has similar properties to those of $L^{s}(R)$, as was shown in [7; Section 6].

For $A, B \in L(R), B$ is called a complement of $A$ if $B \cap A=0$ whereas $C \cap A \neq 0$ for every $C \supset B, C \neq B$. If $B$ is a complement of $A$, then clearly $A+B \in L^{\mathbf{\Delta}}(R)$. Furthermore, if $R^{\mathbf{\Delta}}=0$, then $B \in$ $L^{s}(R)$.

If $A$ is a two-sided ideal of $R$ for which $A \cap A^{l}=0$, then evidently $A^{l}$ is the unique complement of $A$ in $L(R)$. Since $\left(A+A^{l}\right)^{l}=A^{l} \cap A^{l l}$, clearly $A^{l l}$ is the unique complement of $A^{l}$ in case $R^{\mathbf{\Delta}}=0$. In this case, both $A^{l}$ and $A^{l l}$ are in $L^{s}(R)$. By [7; 6.7], $C^{s}(R)=\{A \mid A$ ideal of $\left.R, A \cap A^{l}=0, A=A^{l l}\right\}$ is the center of the lattice $L^{s}(R)$. For each $A \in C^{s}(R)$, it is easily seen that $A^{\mathbf{\Delta}}=0$, that $L^{s}(A)=\left\{B \cap A \mid B \in L^{s}(R)\right\}$, and that $C^{s}(A)=\left\{B \cap A \mid B \in C^{s}(R)\right\}$. Of course, $L^{s}(A) \subset L^{s}(R)$ and $C^{s}(A) \subset C^{s}(R)$.

Every regular ring $R$ has a zero singular ideal. This is evident because $e^{r} \cap e R=0$ for each idempotent $e \in R$. Since $R=e R+e^{r}$, evidently $e R$ and $e^{r}$ are complements of each other and each is in $L^{s}(R)$. Consequently, each principal right ideal $a R \in L^{s}(R)$.

A ring $R$ for which $R^{\mathbf{\Delta}}=0$ and $C^{s}(R)=\{0, R\}$ is called (right) irreducible. An irreducible ring need not be prime. For example, the ring of all $n \times n$ triangular matrices over the ring $Z$ of integers is irreducible by [8; 3.5]. Clearly this ring has a nonzero nilpotent ideal. By $[8 ; 2.1]$, an irreducible ring is prime if and only if it contains no nonzero nilpotent ideal.

If $R$ is a subring of ring $Q$ then $Q$ is called a (right) quotient ring of $R$, and write $R \leqq Q$, if and only if $q R \cap R \neq 0$ each nonzero $q \in Q$. It was proved in [5] that each ring $R$ for which $R^{\mathbf{\Delta}}=0$ has a unique maximal quotient ring $\hat{R}$. By [5; Theorem 2], $\hat{R}$ is a regular ring with unity. Essentially, the definition of $\hat{R}$ in [5] was as follows:

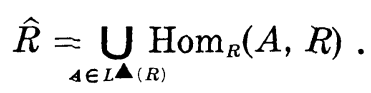

If $x, y \in \hat{R}$, then we take $x=y$ if and only if $x \mathrm{a}=y a$ for every $a$ in some large right ideal $A \subset \operatorname{Dom} x \cap \operatorname{Dom} y$.

In case $R$ is a subring of a ring $Q$, then we may consider $Q$ as a right $R$-module. If we do so, then the assumption $R \leqq Q$ implies that $R \subset^{\prime} Q$, considering $R$ and $Q$ as right $R$-modules. It is easily verified

The more general definition of a quotient ring in [12] and [2] is equivalent to ours in case $R^{\mathbf{\Lambda}}=0$. 
that if $R \leqq Q$ then $Q^{\mathbf{\Delta}}(R)=0$ if and only if $R^{\mathbf{\Delta}}=0$.

2. Some basic lemmas. The rest of this paper will be concerned only with a ring $R$ for which $R^{\mathbf{\Lambda}}=0$. We shall prove in this section that if $Q$ is a quotient ring of such a ring $R$, then the lattices of closed right ideals of $R$ and $Q$ are isomorphic.

2.1 Lemma. If $R \leqq Q$ and $A \in L(Q)$, then $A \in L^{\mathbf{\Delta}}(Q)$ if and only if $A \cap R \in L^{\mathbf{\Delta}}(R)$.

Proof. If $A \in L^{\Delta}(Q)$ and $b \in R, b \neq 0$, then $A \cap b Q \neq 0$ and $a=$ $b q \neq 0$ for some $a \in A$ and $q \in Q$. Now $q C \subset R$ for some $C \in L^{\star}(R)$ by [7;6.1]. Since $Q^{\mathbf{\Delta}}(R)=0, b q C \neq 0$ and therefore $A \cap b R \neq 0$. Hence $(A \cap R) \cap b R \neq 0$ and $A \cap R \in L^{\boldsymbol{\Delta}}(R)$.

On the other hand, let us assume that $A \in L(Q)$ and $A \cap R \in L^{\triangleleft}(R)$. For each nonzero $q \in Q, q C \subset R$ for some $C \in L^{\boldsymbol{\Delta}}(R)$. If we let $B=C \cap$ $(A \cap R)$, then $B \in L^{\boldsymbol{\Delta}}(R)$ and $q B \neq 0$ since $Q^{\mathbf{\Delta}}(R)=0$. Hence $q B \cap(A$ $\cap R) \neq 0$ and we conclude that $q Q \subset A \neq 0$ for each nonzero $q \in Q$. Thus, $A \in L^{\mathbf{\Delta}}(Q)$.

2.2 Lemma. If $R \leqq Q$ and $M$ is a right $Q$-module, then $M$ is a right $R$-module and $M^{\mathbf{\wedge}}(R)=M^{\mathbf{\Delta}}(Q)$.

Proof. If $x \in M$ and $A=x^{r}$ (in $\left.Q\right)$ then $A \in L^{\boldsymbol{\Delta}}(Q)$ if and only if $A \cap$ $R \in L^{\mathbf{\Delta}}(R)$ by 2.1. Therefore, $M^{\mathbf{\Delta}}(R)=M^{\mathbf{\Delta}}(Q)$.

2.3 Corollary. If $R \leqq Q$, then $Q^{\mathbf{\Delta}}=0$.

This follows from 2.2 if we let $M=Q$ and use the assumption that $R^{\mathbf{\Delta}}=0$.

2.4 Lemma. If $R \leqq Q$ and $M$ is a right $Q$-module such that $M^{\wedge}(Q)=0$, then $L^{s}(M, R)=L^{s}(M, Q)$.

Proof. If $A \in L^{s}(M, R)$ and $q \in Q$, then $q B \subset R$ for some $B \in$ $L^{\mathbf{\Delta}}(R)$. Therefore $(A q) B \subset A$ and $A q \subset A$ by [7; 6.4]. Hence, $A \in$ $L(M, Q)$ and we conclude that $L^{s}(M, R) \subset L(M, Q)$.

If $A \in L(M, Q), x \in M$ and $B_{x}=\{b \mid b \in Q, x b \in A\}$, then $x \in A^{s}$ if and only if $B_{x} \in L^{\wedge}(Q)$ by $[7 ; 6.4]$. Therefore, in view of 2.1 , the closure of $A$ relative to $Q$ is the same as its closure relative to $R$. Thus, $L^{s}(M, R)=L^{s}(M, Q)$.

2.5 THEOREM. If $R \leqq Q$, if $M$ is a right $Q$-module for which $M^{\boldsymbol{\wedge}}(Q)=0$ and if $N \in L^{\wedge}(M, R)$, the $L^{s}(M, Q) \cong L^{s}(N, R)$ under the 
correspondence $A \rightarrow A \cap N, A \in L^{s}(M, Q)$.

Proof. By [7; 6.8], $L^{s}(M, R) \cong L^{s}(N, R)$. Thus 2.5 follows from 2.4 .

2.6 CoROLlaRY. If $R \leqq Q$, then $L^{s}(Q) \cong L^{s}(R)$ under the correspondence $A \rightarrow A \cap R, A \in L^{s}(Q)$.

If $R$ is an irreducible ring, so that $C^{s}(R)=\{0, R\}$, then $C^{s}(\hat{R})=$ $\{0, \hat{R}\}$ by 2.6. Hence $\hat{R}$ also is irreducible. Actually, since $\hat{R}$ is regular, $\hat{R}$ is a prime ring by $[8 ; 2.1]$. We state this result as follows.

2.7 Theonem. If $R$ is an irreducible ring, then $\hat{R}$ is a prime ring.

3. $L^{s}(R)$ atomic. Let us assume in this section that $R$ is a ring for which $R^{\mathbf{\Delta}}=0$ and the lattice $L^{s}(R)$ is atomic. We define this to mean that $L^{s}(R)$ has minimal nonzero elements, called atoms, and that each element of $L^{s}(R)$ contains at least one atom. It is proved in [7; 6.9] that a nonzero element $x$ of $R$ is contained in an atom if and only if $x^{r}$ is a maximal element of $L^{s}(R)$. Incidentally, $(x R)^{s}$ is the atom containing $x$.

Two atoms $A$ and $B$ are said to be perspective [1; p. 118], and we write $A \sim B$, if and only if $A$ and $B$ have a common complement. It is easily shown in our case that $A \sim B$ if and only if $A \cup B$ contains a third atom [1; p. 120, Lemma 3]. We proved in [7;6.10] that $A \sim B$ if and only if $a^{r}=b^{r}$ for some nonzero $a \in A$ and $b \in B$. If $A \sim B$ and $B \sim C$ then $a^{r}=b^{r}$ and $b_{1}^{r}=c^{r}$ for some nonzero $a \in A, b, b_{1} \in B$ and $c \in C$. Since $B$ is an atom, $b R \cap b_{1} R \neq 0$ and there exist $x, x_{1} \in R$ such that $b x=b_{1} x_{1} \neq 0$. Hence, $(a x)^{r}=(b x)^{r}=\left(b_{1} x_{1}\right)^{r}=\left(c x_{1}\right)^{r}$. It follows that perspectivity is an equivalence relation on the set of all atoms of $L^{s}(R)$. Clearly for a finite set $\left\{A_{1}, \cdots, A_{n}\right\}$ of perspective atoms, there exist nonzero $a_{i} \in A_{i}$ such that $a_{i}^{r}=a_{j}^{r}$ for each $i$ and $j$.

For each atom $A$ of $L^{s}(R)$, let $A^{*}$ be the union in $L^{s}(R)$ of all atoms perspective to $A$. It is proved in [7] that $A^{*}$ is an ideal of $R$ [7; 6.7] and that $A^{*}$ is an atom of $C^{s}(R)$ [7; 6.12]. Conversely, each atom of $C^{s}(R)$ is of the form $A^{*}$ for some atom $A$ of $L^{s}(R)$.

Since $C^{s}(R)$ is a Boolean algebra, $R$ is the direct union of all atoms of $C^{s}(R)$. Hence, if $\left\{A_{i}^{*}\right.$; $\left.\mathrm{i} \in \Delta\right\}$ is the set of all distinct atoms of $C^{s}(R)$, then the ring-union $S$ of the atoms of $C^{s}(R)$ is a discrete direct sum of these atoms,

$$
S=\sum_{\imath \in \Lambda} A_{\imath}^{*}
$$

Since $S^{l}=0$, evidently $S \leqq R$. Consequently, the maximal quotient 
ring of $R$ is just the maximal quotient ring of $S$.

The following theorem characterizes $\hat{R}$ in terms of left full rings. We shall call a ring $R$ a left full ring if there exists a division ring $D$ and a right $D$-module $M$ such that

$$
R \cong \operatorname{Hom}_{D}(M, M) \text {. }
$$

Evidently we may consider $M$ as a $(R, D)$-module.

3.1 THEOREM. If $R$ is a right irreducible ring, then $\hat{R}$ is a left full ring. If $R$ is right reducible, then $\hat{R}$ is a complete direct sum of left full rings.

Proof. Consider first the case in which $R$ is irreducible. Since $\hat{R}$ is regular and $L^{s}(R) \cong L^{s}(\hat{R})$, the lattice $L^{s}(\hat{R})$ is atomic and its atoms are principal and hence minimal right ideals of $\hat{R}$. Since $\hat{R}$ is prime and has minimal right ideals, it is primitive. Let $e$ be an idempotent element of $\hat{R}$ such that $e \hat{R}$ is a minimal right ideal. Then $M=\hat{R} e$ is a minimal left ideal of $\hat{R}$ and $D=e \hat{R} e$ is a division ring. Since $x \hat{R} e \neq 0$ for each nonzero $x \in \hat{R}$ by the primeness of $\hat{R}$, evidently $\hat{R}$ is a right quotient ring of $M$. However, $\hat{R}$ is a maximal right quotient ring so that we must have $\hat{M}=\hat{R}$. Besides being a ring, $M$ may be considered to be a $(\hat{R}, D)$-module. Clearly the right ideals of $M$ are its $D$-submodules. Thus, $M$ is the only large right ideal of $\boldsymbol{M}$. Consequently,

$$
\operatorname{Hom}_{M}(M, M) \text {, }
$$

considering $M$ as a right $M$-module, is the maximal right quotient ring of $M$. Since $x(a e)=x(e a e)$ for each $x \in M$ and $a \in \hat{R}$, evidently

$$
\operatorname{Hom}_{M}(M, M)=\operatorname{Hom}_{D}(M, M) \text {. }
$$

Since $\hat{M}=\hat{R}$, this proves that $\hat{R}$ is a left full ring.

If $R$ is not irreducible, then there exists a set $\left\{R_{i} ; i \in \Delta\right\}$ of irreducible rings, each having an atomic lattice of closed right ideals, such that

$$
\sum_{i \in \Lambda} R_{i} \leqq R
$$

by our previous results. We shall not give the details, but it is easily seen that if

$$
S=\sum_{i \in A} R_{i}, \text { then } \hat{S}=\sum_{i \in A}^{\prime} \hat{R}_{i}
$$

where $\Sigma^{\prime}$ designates the complete direct sum. Since $\hat{S}=\hat{R}$, this proves the second part of 3.1 . 
The important special case of this theorem when $R$ is a primitive ring was proved by Utumi [12; 5.1] and Wong [13; 4.1]. Both Utumi and Lambek [10] have independently proved the theorem if $R$ is prime.

4. $L_{s}(R)$ finite-dimensional. The usual assumption that $R^{\mathbf{\Lambda}}=0$ is made for each ring $R$ of this section. If either the a.c.c. or the d.c.c. holds for $L^{s}(R)$ then so does the other one. In fact, each is equivalent to the assumption that $L^{s}(R)$ contains a maximal chain of finite length. When this condition is satisfied, a dimension function $d$ may be defined on $L^{s}(R)$ as follows [1; p. 67]: for each $A \in L^{s}(R), d(A)$ is the length of the longest chain joining 0 to $A$. Incidentally, every maximal chain joining 0 to $A$ has the same length $d(A)$. We shall assume in this section that such a dimension function $d$ is defined on $L^{s}(R)$ and that $d(R)$ is finite. Since the lattice $L^{s}(R)$ is also complemented, each $A \in$ $L^{s}(R)$ is a direct union of $d(A)$ atoms $[1 ;$ p. 105].

It is proved in [9; 3.4] that if $d(R)$ is finite then for each $a \in R$, $a R \in L^{\boldsymbol{\Delta}}(R)$ if and only if $a^{r}=0$. Of course, $a^{l}=0$ whenever $a R \in L^{\boldsymbol{\Lambda}}(R)$. Thus, $D(R)=\left\{a \mid a \in R, a R \in L^{\boldsymbol{\Delta}}(R)\right\}$ is the set of regular elements of $R$. Each $a \in D(R)$ has an inverse in $\hat{R}$. For, by the regularity or $\hat{R}$, $(a b-1) a=a(b a-1)=0$ for some $b \in \hat{R}$. Since $(a b-1)^{r} \supset a R$, a large element of $L^{\Delta}(R), a b-1=0$ in view of 2.1 and 2.3. Also, $b a-1=0$ since $a^{r}=0$ in $\hat{R}$ as well as in $R$. Consequently, $b=\mathrm{a}^{-1}$.

4.1 Theorem. If $R$ is irreducible and $d(R)=n$, then $\hat{R}$ is a full ring of dimension $n$.

By a full ring of dimension $n$ we mean a ring isomorphic to $\operatorname{Hom}_{D}(M, M)$ where $D$ is a division ring and $M$ is a right $D$-module of dimension $n$.

If we select $M=\hat{R} e$ as in the proof of 3.1 , then $M \leqq \hat{R}$ and the lattices $L^{s}(R), L^{s}(M)$ and $L^{s}(\hat{R})$ are isomorphic by 2.6. Since the right ideals of $M$ are its $D$-submodules, $M$ is an $n$-dimensional vector space over $D$. Hence 4.1 follows from 3.1 .

A different proof of 4.1 was given in $[9 ; 3.6]$.

If $R$ is a prime ring for which $d(R)$ is finite, then it was proved in [3; Theorem 10] and in [9; 3.5] that every large right ideal of $R$ contains a regular element. Since $B=\{b \mid b \in R, q b \in R\}$ is a large right ideal of $R$ for each $q \in \hat{R}$, clearly $q b=\alpha$ for some $b \in D(R)$ and $a \in R$; that is, $q=a b^{-1}$. This proves the following theorem of Goldie $e^{2}$ [3] (also proved in [11] and [9]).

${ }^{2}$ That each ring considered by Goldie has a zero singular ideal is proved in $[4 ; 3.2]$. 
4.2 THeOREm. If $R$ is a prime ring for which $d(R)=n$, then not only is $\hat{R}$ the full ring of linear transformations of an $n$-dimensional vector space over a division ring but also $R=\left\{a b^{-1} \mid a \in R, b \in D(R)\right\}$.

From 3.1 and 4.1, we easily deduce the following theorem.

4.3 Theorem. If $R$ is a ring for which $d(R)$ is finite, then $\hat{R}$ is a direct sum of a finite number of finite-dimensional full rings.

A ring $R$ is called semiprime if it contains no nonzero nilpotent ideal. We recall that if $S$ is the direct sum of the atoms of $C^{s}(R)$, then $S \leqq R$. Since each nonzero ideal of $R$ has nonzero intersection with some atom of $C^{s}(R)$, evidently $R$ is semiprime if and only if each atom of $C^{s}(R)$ is prime. The following theorem was recently proved by Goldie [4].

4.4 Theorem. If $R$ is a semiprime ring for which $d(R)$ is finite, then not only is $\hat{R}$ a direct sum of a finite number of finite-dimensional full rings but also $R=\left\{a b^{-1} \mid a \in R, b \in D(R)\right\}$.

The first part of 4.4 follows directly from 4.3. To prove the second part, let $S=R_{1} \oplus \cdots \oplus R_{k}$ be the sum of the atoms of $C^{s}(R)$. Then $\hat{R}=\hat{S}=\hat{R}_{1} \oplus \cdots \oplus \hat{R}_{k}$. If $q_{i} \in \hat{R}$, then $q_{i}=a_{i} b_{i}^{-1}$ for some $a_{i} \in R_{i}$ and $b_{i} \in D\left(R_{i}\right)$ by 4.2. Thus, if $q=q_{1}+\cdots+q_{k}, a=a_{1}+\cdots+a_{k}$, and $b=b_{1}+\cdots+b_{k}, q=a b^{-1}$. This proves the second part of 4.4.

A converse of 4.4 has been given by Goldie [5; 4.4]. He proved that if $R$ is a ring for which $d(\hat{R})$ is finite and $\hat{R}=\left\{a b^{-1} \mid a \in R, b \in\right.$ $D(R)$, then $R$ is semiprime. Naturally, this implies the following converse of 4.2: If $R$ is a ring for which $\hat{R}$ is a finite-dimensional full ring and $\hat{R}=\left\{a b^{-1} \mid a \in R, b \in D(R)\right\}$ then $R$ is prime.

\section{BIBLIOGRAPHY}

1. G. Birkhoff, Lattice theory, Amer. Math. Soc. Colloquium Publications, rev. ed. vol. 25. New York, 1948.

2. G. D. Findlay and J. Lambek. A generalized ring of quotient, I and II, Can. Math, Bull., 1 (1958), 77-85, 155-167.

3. A. W. Goldie, The structure of prime rings under ascending chain conditions, Proc. London Math. Soc., 8 (1958), 589-608.

4. - Semi-prime rings with maximum condition, ibid 10 (1960), 201-220.

5. R. E. Johnson, The extended centralizer of a ring over a module, Proc. Amer. Math. Soc., 2 (1951), 891-895.

6. - Structure theory of faithful rings I. Closure operations on lattices, Trans. Amer. Math. Soc., 84 (1957), 508-522.

7. — II. Restricted rings, ibid, p.p. 523-544.

8. - III. Irreducible rings, Proc. Amer. Math. Soc., 11 (1960), 710-717. 
9. R. E. Johnson and E. T. Wong, Quasi-injective modules and irreducible rings, J. London Math. Soc., 36 (1961), 260-268.

10. J. Lambek, On the structure of semi-prime rings and theirs of quotients, Can. J. Math. 13(1961), 392-417.

11. L. Lesieur and R. Croisot, Sur les anneaux premiers noethériéns á gauche, Ann. Sci. Ec. Norm. Sup., 76 (1959), 161-183.

12. Y. Utumi, On quotient rings, Osaka Math. J., 8 (1956), 1-18.

13. E. T. Wong, Quotient rings, Ph. D. Thesis, U. of Rochester (1956).

14. E. T. Wong and R. E. Johnson, Self-injective rings, Can. Math. Bull., 2 (1959), 167173.

UNIVERSITY OF ROCHESTER 


\section{PACIFIC JOURNAL OF MATHEMATICS}

\section{EDITORS}

\author{
RaLPh S. Phillips \\ Stanford University \\ Stanford, California \\ F. H. BRowNELL \\ University of Washington \\ Seattle 5 , Washington
}

A. L. Whiteman

University of Southern California

Los Angeles 7, California

L. J. Paige

University of California

Los Angeles 24, California

\author{
E. F. BECKENBACH \\ T. M. CHERRY
}

\author{
ASSOCIATE EDITORS

$\begin{array}{lll}\text { D. DERRY } & \text { H. L. ROYDEN } & \text { E. G. STRAUS } \\ \text { M. OHTSUKA } & \text { E. SPANIER } & \text { F. WOLF }\end{array}$

\section{SUPPORTING INSTITUTIONS}

\author{
UNIVERSITY OF BRITISH COLUMBIA \\ CALIFORNIA INSTITUTE OF TECHNOLOGY \\ UNIVERSITY OF CALIFORNIA \\ MONTANA STATE UNIVERSITY \\ UNIVERSITY OF NEVADA \\ NEW MEXICO STATE UNIVERSITY \\ OREGON STATE COLLEGE \\ UNIVERSITY OF OREGON \\ OSAKA UNIVERSITY \\ UNIVERSITY OF SOUTHERN CALIFORNIA
}

\author{
STANFORD UNIVERSITY \\ UNIVERSITY OF TOKYO \\ UNIVERSITY OF UTAH \\ WASHINGTON STATE COLLEGE \\ UNIVERSITY OF WASHINGTON \\ AMERICAN MATHEMATICAL SOCIETY \\ CALIFORNIA RESEARCH CORPORATION \\ HUGHES AIRCRAFT COMPANY \\ SPACE TECHNOLOGY LABORATORIES \\ NAVAL ORDNANCE TEST STATION
}

Mathematical papers intended for publication in the Pacific Journal of Mathematics should be typewritten (double spaced), and the author should keep a complete copy. Manuscripts may be sent to any one of the four editors. All other communications to the editors should be addressed to the managing editor, L. J. Paige at the University of California, Los Angeles 24, California.

50 reprints per author of each article are furnished free of charge; additional copies may be obtained at cost in multiples of 50 .

The Pacific Journal of Mathematics is published quarterly, in March, June, September, and December. The price per volume (4 numbers) is $\$ 12.00$; single issues, $\$ 3.50$. Back numbers are available. Special price to individual faculty members of supporting institutions and to individual members of the American Mathematical Society: $\$ 4.00$ per volume; single issues, $\$ 1.25$.

Subscriptions, orders for back numbers, and changes of address should be sent to Pacific Journal of Mathematics, 103 Highland Boulevard, Berkeley 8, California.

Printed at Kokusai Bunken Insatsusha (International Academic Printing Co., Ltd.), No. 6, 2-chome, Fujimi-cho, Chiyoda-ku, Tokyo, Japan.

\section{PUBLISHED BY PACIFIC JOURNAL OF MATHEMATICS, A NON-PROFIT CORPORATION}

The Supporting Institutions listed above contribute to the cost of publication of this Journal, but they are not owners or publishers and have no responsibility for its content or policies.

Reprinted 1966 in the United States of America 


\section{Pacific Journal of Mathematics}

\section{Vol. 11, No. 4}

A. V. Balakrishnan, Prediction theory for Markoff processes . . . . . . . . . . 1171

Dallas O. Banks, Upper bounds for the eigenvalues of some vibrating systems . . . . 1183

A. Białynicki-Birula, On the field of rational functions of algebraic groups ...... 1205

Thomas Andrew Brown, Simple paths on convex polyhedra .............. 1211

L. Carlitz, Some congruences for the Bell polynomials . . . . . . . . . . . . 1215

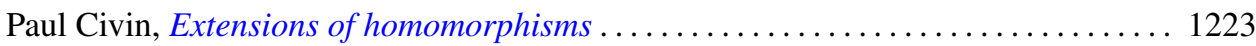

Paul Joseph Cohen and Milton Lees, Asymptotic decay of solutions of differential

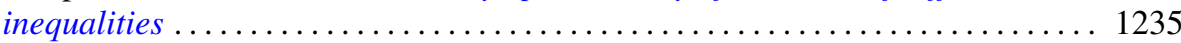

István Fáry, Self-intersection of a sphere on a complex quadric . . . . . . . . . . 1251

Walter Feit and John Griggs Thompson, Groups which have a faithful representation

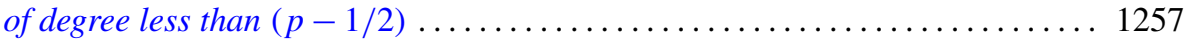

William James Firey, Mean cross-section measures of harmonic means of convex

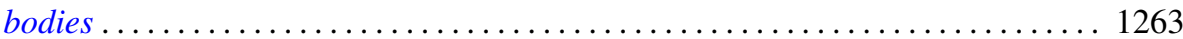

Avner Friedman, The wave equation for differential forms . . . . . . . . . . 1267

Bernard Russel Gelbaum and Jesus Gil De Lamadrid, Bases of tensor products of

Banach spaces ................................... 1281

Ronald Kay Getoor, Infinitely divisible probabilities on the hyperbolic plane . . . . 1287

Basil Gordon, Sequences in groups with distinct partial products . . . . . . . . . . . . 1309

Magnus R. Hestenes, Relative self-adjoint operators in Hilbert space . . . . . . . . . 1315

Fu Cheng Hsiang, On a theorem of Fejér ......................... 1359

John McCormick Irwin and Elbert A. Walker, On N-high subgroups of Abelian

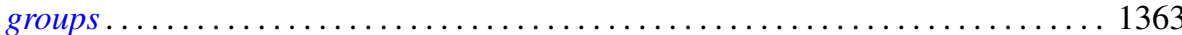

John McCormick Irwin, High subgroups of Abelian torsion groups . . . . . . . . . 1375

R. E. Johnson, Quotient rings of rings with zero singular ideal . . . . . . . . . . . 1385

David G. Kendall and John Leonard Mott, The asymptotic distribution of the time-to-escape for comets strongly bound to the solar system ...

Kurt Kreith, The spectrum of singular self-adjoint elliptic operators ....

Lionello Lombardi, The semicontinuity of the most general integral of the calculus of variations in non-parametric form ................................

Albert W. Marshall and Ingram Olkin, Game theoretic proof that Chebyshev inequalities are sharp

Wallace Smith Martindale, III, Primitive algebras with involution . . William H. Mills, Decomposition of holomorphs ..............

James Donald Monk, On the representation theory for cylindric algebras . . . . . . 1447

Shu-Teh Chen Moy, A note on generalizations of Shannon-McMillan theorem . . . . 1459

Donald Earl Myers, An imbedding space for Schwartz distributions . .

John R. Myhill, Category methods in recursion theory .........

Paul Adrian Nickel, On extremal properties for annular radial and circular slit mappings of bordered Riemann surfaces

Edward Scott O'Keefe, Primal clusters of two-element algebras . .

Nelson Onuchic, Applications of the topological method of Wazewski to certain

problems of asymptotic behavior in ordinary differential equations ...

Peter Perkins, A theorem on regular matrices................

Clinton M. Petty, Centroid surfaces .... 